\title{
A serological prevalence survey of Brucella abortus in cattle of rural communities in the province of KwaZulu-Natal, South Africa
}

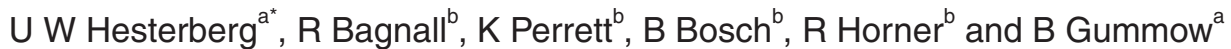

\begin{abstract}
A serological survey of Brucella abortus in cattle originating from communal grazing areas of Kwa Zulu Natal was carried out between March 2001 and December 2003. The survey was designed as a 2-stage survey, considering the diptank as the primary sampling unit. In total 46025 animals from 446 diptanks of 33 magisterial districts were sampled and tested using the Rose Bengal test and Complement Fixation Test. The apparent prevalence at district level was adjusted for clustering, diagnostic test sensitivity and specificity, and mapped using ArcView version 3.3. The prevalence of brucellosis in communal grazing areas of Kwa-Zulu Natal was found to be $1.45 \%(0.84-2.21 \%)$ and varied from 0 to $15.6 \%$ between magisterial districts. In 19 of the 33 magisterial districts no serological reactors were observed. A large variation in prevalence was found within diptank areas. Brucellosis was found to be most prevalent in the northeastern area of the province. The findings of the survey are discussed.
\end{abstract}

Key words: Africa, Brucella abortus, brucellosis, communities, prevalence, rural, zoonosis. Hesterberg U W, Bagnall R, Perrett K, Bosch B, Horner R, Gummow B A serological prevalence survey of Brucella abortus in cattle of rural communities in the province of KwaZulu-Natal, South Africa. Journal of the South African Veterinary Association (2008) 79(1): 15-18 (En.). Section of Epidemiology, Department of Production Animal Studies, Faculty of Veterinary Science, University of Pretoria, Private Bag X04, Onderstepoort, 0110 South Africa.

\section{INTRODUCTION}

Brucellosis is a disease caused by members of the genus Brucella, a facultative intracellular parasite, affecting many animal species and humans. In bovines the disease is caused by Brucella abortus and is characterised by abortion in late pregnancy and a successive possibility of infertility in females. Brucellosis has long been known to be present in South Africa and from 1996 to 2004 between 291 and 457 outbreaks of bovine brucellosis were reported yearly to the $\mathrm{OIE}^{14}$. The disease is known to have a high prevalence in southern Africa, especially in intensively farmed areas and has a strong economic impact. In a scenario where $14.7 \%$ of herds were infected in 1990, losses to farmers were estimated to be around 300 rand million annually ${ }^{8}$. However, cattle from remote rural villages were found in 1 study to have a much lower

asection of Epidemiology, Department of Production Animal Studies, Faculty of Veterinary Science, University of Pretoria, Private Bag X04, Onderstepoort, 0110 South Africa.

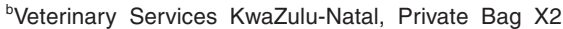
Cascades, 3202 South Africa.

Author for correspondence. Present address: Centre for Epidemiology and Risk Analysis, Veterinary Laboratories Agency, Woodham Lane, Addlestone, KT15 3NB UK. E-mail: u.hesterberg @ vla.defra.gsi.gov.uk Received: July 2007. Accepted: January 2008. sero-prevalence than cattle from periurban areas ${ }^{1}$.

Besides the impact the disease has on livestock, brucellosis is also an important zoonosis, more commonly known as undulant fever. Worldwide around 500000 persons are estimated to be affected by brucellosis annually ${ }^{16}$. The established mode of transmission of Brucella spp. to humans is usually by
Fig. 1: Location of the province of KwaZulu-Natal. direct contact with infected animals or their carcasses or through ingestion of infected unpasteurised milk or dairy products $^{4,16}$. The former homelands of South Africa are generally regarded as resource-poor areas with a weak infrastructure, a high unemployment rate and subsistence farming dominating other agricultural activities. Production indicators, such as herd mortality and calving rate, are generally much below the results of commercial farmers ${ }^{12}$. Very little is known about the prevalence of important zoonotic and production diseases of cattle in these areas, which is essential information for the prioritisation and implementation of disease control schemes.

Besides this, the public awareness of zoonotic diseases such as brucellosis is very low in southern Africa and a lack of sufficient knowledge of the disease among physicians in addition to the absence of effective prevention and management strategies have contributed to the wide spread of the disease, but the actual rate of infections in humans is unknown ${ }^{8}$. To gain an understanding of the prevalence of brucellosis in the rural livestock population, the Provincial Veterinary Services of KwaZulu-Natal, a coastal South African province (Fig. 1), initiated a largescale serological survey, the results of which are presented and discussed here.

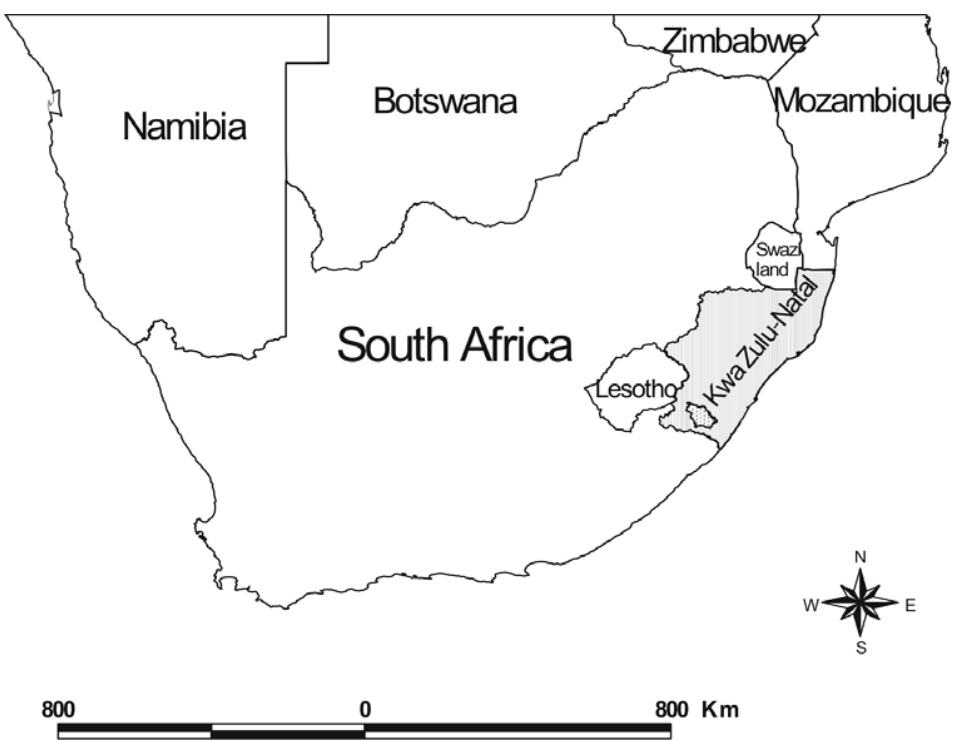




\section{MATERIALS AND METHODS}

Serological samples were taken from March 2001 until December 2003. At the time of the survey the province of KwaZulu-Natal was divided into 52 magisterial districts according to the existing political boundaries. This division was used in the design of the survey and required sample sizes were calculated on a district basis. Since rural communities are not present in all the districts, only 33 districts were included. The sample sizes and strategy was developed in consultation with a statistician (B. Williams, WHO, Geneva). Diptanks were the primary sampling unit and approximately 1500 cattle were sampled in each district at between 4 and 27 diptanks depending on the number of diptanks present in the district. Diptanks were selected on a random basis by drawing numbers out of a hat. The sampling design intended that animals were also selected on a random basis provided their owners approved of the collection of samples, which the large majority (>80\%) did. Samples were only taken from fully-grown animals with no stratification according to age or sex. Animal Health Technicians bled the cattle according to standard procedures from the jugular vein into serum tubes and returned the serum samples to Allerton Laboratory in Pietermaritzburg weekly for screening with the Rose Bengal test $(\mathrm{RBT})^{9}$. Positive samples were retested with the Complement Fixation test $(\mathrm{CFT})^{10}$ and if a sample remained positive on this test it was interpreted as positive.

Once all samples had been tested, the results were entered into a spreadsheet (Excel 97; Microsoft Corporation, USA) and a database program (Access 97; Microsoft Corporation, USA). Results at diptank level were cross-referenced with a database on the geographic location of diptanks, which had been collated by the State Veterinary Services in KwaZuluNatal. For the creation of diptank maps only diptanks that could be matched with certainty in the GIS database were used. All maps were created in ArcView 3.3 (ESRI, Redlands, California, USA).

For districts where serological reactions were found, the apparent prevalence was adjusted for the combined diagnostic test sensitivity and specificity (Table 1) according to Rogan and Gladen ${ }^{17}$. To account for the clustering effect of sampling at diptanks, the $95 \%$ confidence intervals around the mean true prevalence were adjusted according to Thrusfield ${ }^{20}$. To allow for uncertainty about the test sensitivities, these were entered as a probability distribution (Table 1) and the above described parameters were obtained from the output of a simulation with 10000

Table 1: Distribution functions used to estimate the diagnostic test sensitivity and specificity for the adjustment of the apparent prevalence.

\begin{tabular}{ll}
\hline Parameter & Probability distribution $^{(\text {reference) }}$ \\
\hline Sensitivity CFT (Se1): & Beta $(61,13)^{15}$ \\
Sensitivity RBT (Se2) & Triangular $(0.688,0.81,0.936)^{7}$ \\
Combined sensitivity & $($ Se1xSe2) \\
Specificity RBT & Beta $(1052,1)^{19}$ \\
\hline
\end{tabular}

iterations, using the mean for the true prevalence and the relevant confidence intervals for the $95 \%$ confidence interval of the prevalence.

For districts where no serological reactors were recorded, calculations were carried out using the program Survey Tool-box ${ }^{3}$ to evaluate how certain one can be about the absence of the disease. The software allows for integration of test sensitivity and specificity, which were entered as the mean of the distributions in Table 1, and uses a hypergeometric distribution function for assessing freedom of disease. These calculations were done assuming a minimum prevalence of $1 \%$ brucellosis and a Types I and II errors of $5 \%$.

\section{RESULTS}

The number of diptanks sampled and the number of cattle sampled in each district are given in Fig. 2.

The mean provincial prevalence of brucellosis in the communal areas of KwaZulu-Natal was found to be $1.45 \%$ (95\% $\mathrm{LCL}=0.84 ; 95 \% \mathrm{UCL}=2.21 \%)$. The mean adjusted prevalence for each district and diptank are given in Figs 3 and 4, respectively.

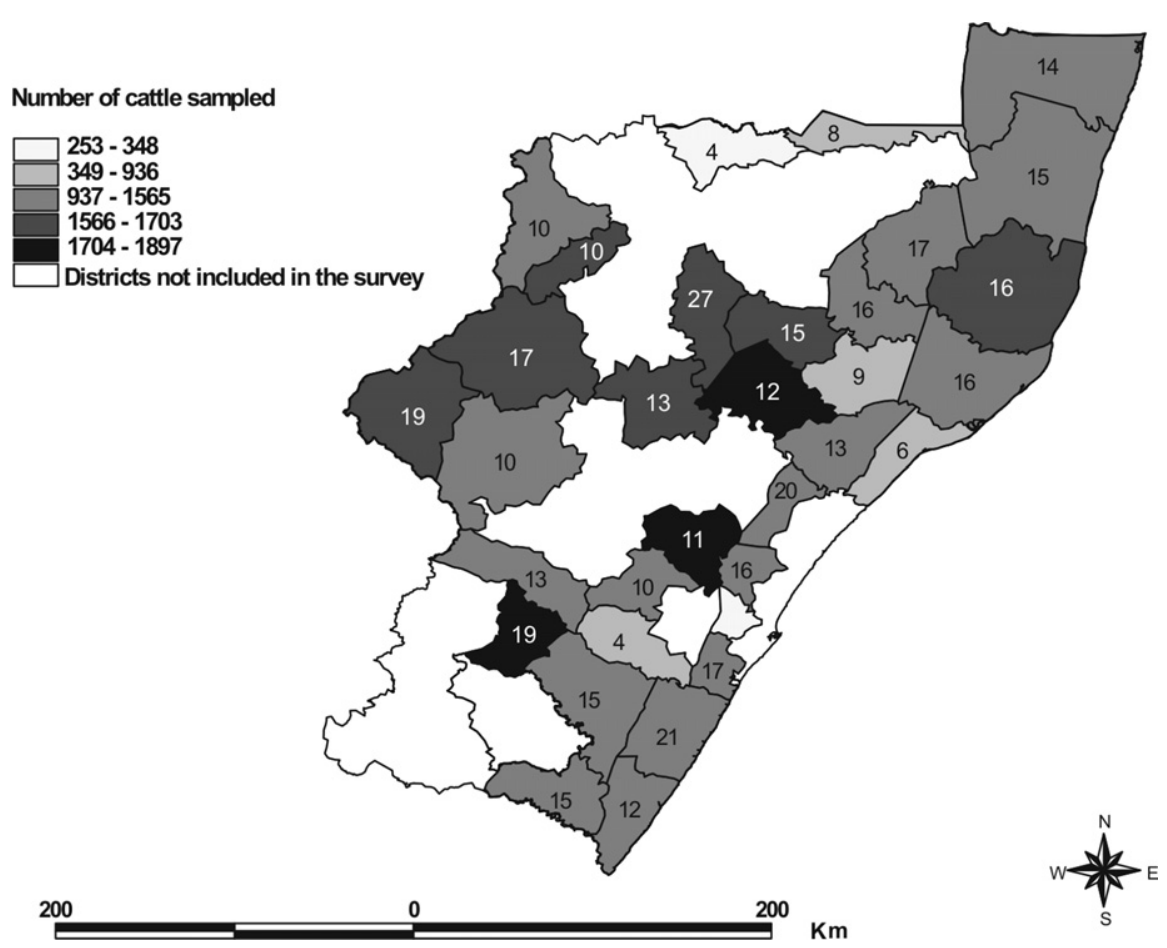

Fig. 2: Number of animals (shading) and diptanks sampled (figures) for bovine brucellosis in a serological survey of KwaZulu-Natal in 2001-2003.
For 19 districts no serological reactions were recorded (Table 2). With the exception of 2 districts where the sample sizes were too low (Pinetown and Paul Pietersburg), one can be $99 \%$ confident that in these districts the serological prevalence is below $1 \%$.

\section{DISCUSSION}

The serological prevalence of brucellosis in most of the districts included in this survey was below $1 \%$ with a $99 \%$ confidence. Considering that no formal control programme is in place, that only very few vaccinations are carried out each year, and that other surveys in sub-Saharan Africa frequently encountered a prevalence in excess of $10 \%{ }^{11}$, the low prevalence or even absence of the disease here, is surprising, although a previous study in a similar South African rural setting also found a comparably low result ${ }^{2}$. In the infected districts in the northeastern parts, the mean prevalence ranged from $2.4 \%$ to $15.5 \%$ and this higher prevalence is supported by fairly frequent reports of abortions and additional unpublished serological evidence from the area (KZN Veterinary Services, pers. comm., 2006). 


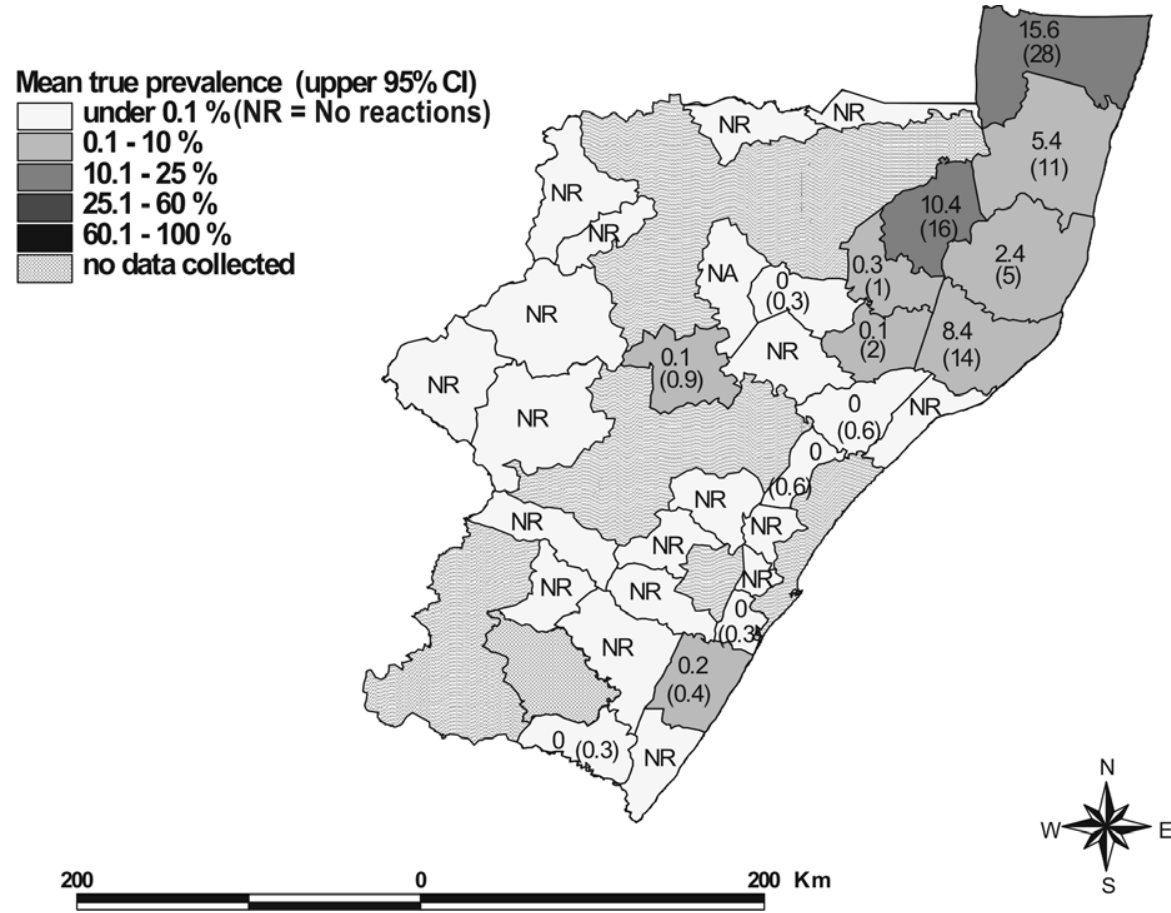

Fig. 3: Mean true prevalence (shading) and upper $95 \%$ confidence interval (values in brackets) of Brucella abortus in communal areas of KwaZulu-Natal surveyed in 2001-2003.

Similar clinical observations, such as abortions, hygromas and infertility, were made under comparable circumstances by McDermott \& Arimi ${ }^{11}$.

Brucellosis was known to occur in the commercial herds in many areas of KwaZulu-Natal, but was thought to have been eradicated by intensive vaccination and test and slaughter programmes car- ried out in the past. While in the northeastern area beef and game farming predominate, dairy farming prevails in occurs in the western parts of the province. Commercial dairy cattle are generally tested on a monthly basis and farmers usually follow a vaccination and control strategy, but commercial beef cattle are the southeastern area and a mix of the 2

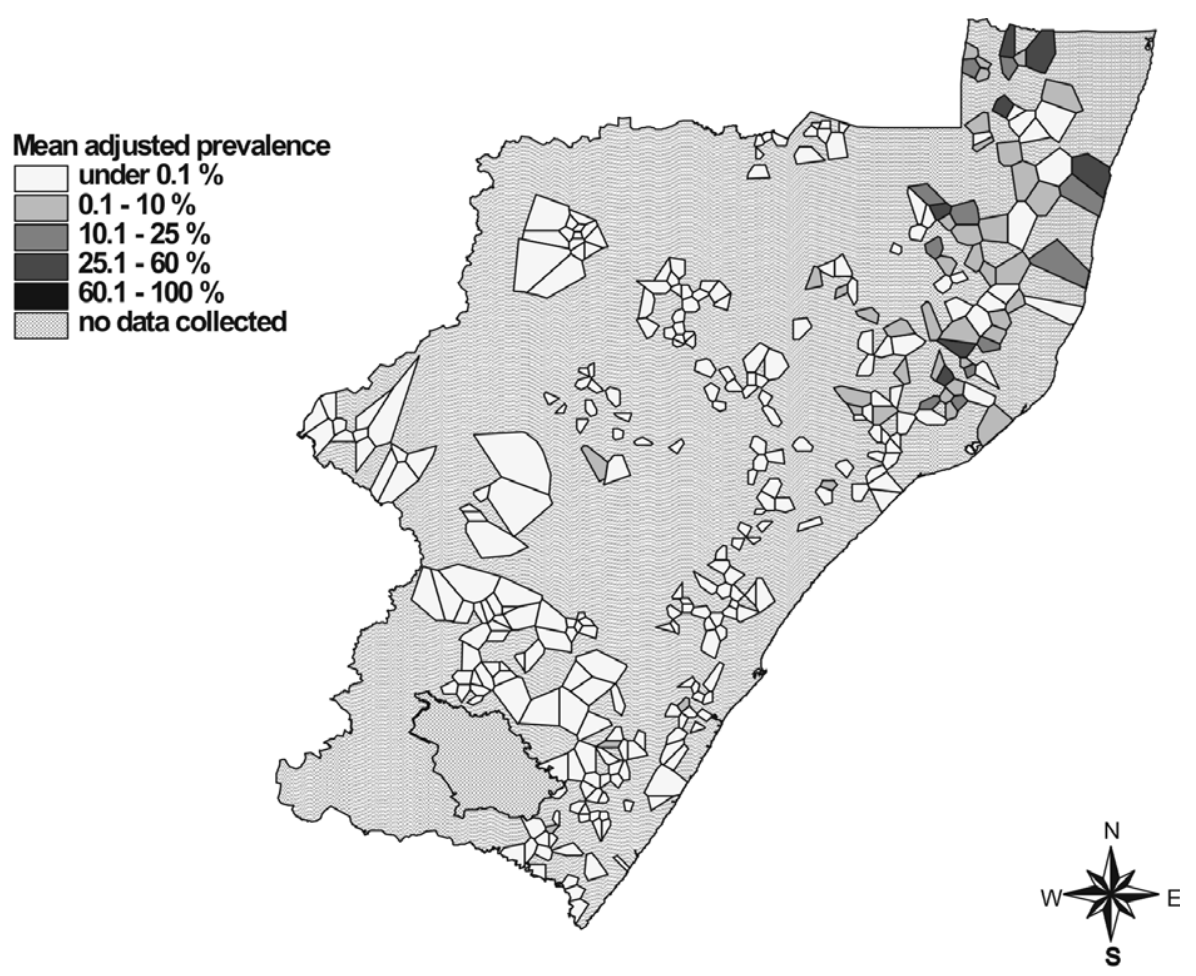

200 0 $200 \mathrm{Km}$

Fig. 4: Mean true prevalence of Brucella abortus in communal cattle based on diptank surveys during the period 2001-2003 in KwaZulu-Natal, South Africa.

tested at most every 5 years and consequently the brucellosis status is much more uncertain and far less controlled in beef-farming areas. As owners in rural communities generally do not sell their cattle, surrounding commercial farms are the main source of new stock. Purchase of infected stock is an important risk factor for the introduction of the disease $\mathrm{s}^{6,18}$ and this practice combined with the much larger testing intervals in beef herds could possibly account for the occurrence of brucellosis in the northeastern parts of the country.

Another known risk factor with regard to between-herd transmission is the proximity to infected herds and grazing patterns that allow sharing of pastures with infected herds.

Cattle in the rural areas in KwaZuluNatal are generally grazed on communal pastures and can move over distances of several kilometres which could lead to contamination of large areas as calving is not restricted to a specific place, such as a pen. This could function as a source of infection for other groups of cattle within that community utilising the same pasture, a factor that has been found to be important in the risk of infection in Zambia ${ }^{13}$. The practice of extensive grazing should however, reduce the infection rate. Although, in general, the contact of herds from different diptanks is thought to be limited, in the findings of this survey, many infected diptanks are adjacent to other infected diptanks. However, several diptanks do also have uninfected neighbours and it is impossible to differentiate from the results of this survey if neighbouring infections are caused by disease spread or multiple introductions of disease through purchase of infected animals.

At a small number of diptanks outside the northeastern area serological reactions occurred, albeit to very limited extent. These reactions could either represent false positives not accounted for in the adjustment for the specificity of the test, or the presence of the disease in an initial phase and would need to be followed up by further investigation to allow a differentiation. At present, if a serological reaction is found, the case is not explored further, primarily due to technical difficulties with regard to the identification of individual animals and the absence of a control and compensation scheme for detected cases.

Although a rapid spread of the disease from the affected area is not very likely, because cattle are not often traded or moved over large distances, and the actual importance of brucellosis compared to other impacts on production parameters 
Table 2: Seronegative districts and the probability of presence of brucellosis at a prevalence of above $1 \%$ given the sample size.

\begin{tabular}{lrrr}
\hline District & Population size & Sample size & Probability \\
\hline Bergville & 32105 & 1650 & $<0.01$ \\
Dannhauser & 11054 & 1631 & $<0.01$ \\
Estcourt & 25118 & 1500 & $<0.01$ \\
Ixopo & 29580 & 1500 & $<0.01$ \\
Klip River & 47730 & 1672 & $<0.01$ \\
Mpendele & 24433 & 1300 & $<0.01$ \\
Mtunzini & 9462 & 809 & $<0.01$ \\
Ndwedwe & 20645 & 1504 & $<0.01$ \\
Newcastle & 12366 & 1544 & $<0.01$ \\
New Hannover & 11000 & 1742 & $<0.01$ \\
Nkandla & 78520 & 1770 & $<0.01$ \\
Nqutu & 44488 & 1605 & $<0.01$ \\
Paul Pietersburg & 15300 & 348 & 0.07 \\
Pietermaritzburg & 17734 & 1565 & $<0.01$ \\
Pinetown & 7000 & 253 & 0.14 \\
Polela & 38275 & 1897 & $<0.01$ \\
Port Shepstone & 12124 & 1503 & $<0.01$ \\
Richmond & 1336 & 616 & $<0.01$ \\
Simdlangentsha & 8000 & 710 & $<0.01$ \\
\hline
\end{tabular}

such as calving percentage might be limited $^{12}$, the implementation of a control strategy for brucellosis is advisable, mainly because of the zoonotic potential of the disease. An attempt could be made to decrease the impact of the disease on the local population through awareness campaigns. This approach could be initiated through training of animal health technicians on the routes of infection and preventive measures such as boiling of milk before consumption and avoidance of contact with aborted material and placentas. Owing to the relatively nonspecific symptoms in humans and a frequent lack of information on zoonotic diseases ${ }^{5}$, it is further important to inform and collaborate with the human health services to increase the likelihood of correct diagnosis and treatment as well as to advocate the prevention of the disease through precautionary measures.

\section{ACKNOWLEDGEMENTS}

The authors thank the staff of KZN Veterinary Services involved in this survey and the stock owners for their cooperation. Without their assistance the work could not have been carried out. This project was approved by the Research and Animal Use and Care committees of the Faculty of Veterinary Science, Univer- sity of Pretoria and registered as protocol no. V058/04

\section{REFERENCES}

1. Bakunzi F R., Zyambo G C, Morris S D 1993 A serological survey of bovine brucellosis in the Molopo District of Bophuthatswana. Journal of the South African Veterinary Association 64: 154-155

2. Bishop G C 1984 A brucellosis serological survey on beef cattle slaughtered at Cato Ridge Abattoir. Journal of the South African Veterinary Association 55: 185-186

3. Cameron A R 1999 Survey Toolbox - A practical manual and software package for active surveillance of livestock diseases in developing countries. Australian Centre for International Agricultural Research Monograph No. 54

4. Chomel B B, DeBess E E, Mangiamele D M, Reilly K F, Farver T B, Sun R K Barrett L R 1994 Changing trends in the epidemiology of human brucellosis in California from 1973 to 1992: a shift toward foodborne transmission. Journal of Infectious Diseases 170: 1216-1223

5. Coulibaly N D, Yameogo K R 2000 Prevalence and control of zoonotic diseases: collaboration between public health workers and veterinarians in Burkina Faso. Acta

6. Crawford R P, Huber J D, Adams B S 1990. Epidemiology and surveillance. In Nielsen K, Duncan J R Animal brucellosis. CRC Press, Boston, USA: 131-152

7. Gall D, Nielsen K 2004 Serological diagnosis of bovine brucellosis: a review of test Tropica 76: 53-57 performance and cost comparison. Review of Science and Technology 23: 989-1002

8. Godfroid J, Bosman P P, Herr S, Bishop G C 2004 Bovine brucellosis. In Coetzer J A W, Tustin R C Infectious Diseases of Livestock. Oxford University Press, Cape Town: 1510 1527

9. Herr S, Bishop G, Bolton T F W, van der Merwe D 1979 Onderstepoort Brucellosis Serology Laboratory Manual. Onderstepoort Veterinary Institute, Onderstepoort

10. Herr S, Huchzermeyer H F, Te Brugge L A, Williamson C C, Roos J A, Schiele G J 1985 The use of a single complement fixation test technique in bovine brucellosis, Johne's disease, dourine, equine piroplasmosis and $\mathrm{Q}$ fever serology. Onderstepoort Journal of Veterinary Research 52: 279-82

11. McDermott J J, Arimi S M 2002 Brucellosis in sub-Saharan Africa: epidemiology, control and impact. Veterinary Microbiology 90: 111134

12. Mokantla E, McCrindle C M, Sebei J P Owen R 2004 An investigation into the causes of low calving percentage in communally grazed cattle in Jericho, North West Province. Journal of the South African Veterinary Association 75: 30-36

13. Muma J B, Samui K L, Siamudaala V M, Oloya J, Matop G, Omer MK, Munyeme M, Mubita C, Skjerve E 2006 Prevalence of antibodies to Brucella spp. and individual risk factors of infection in traditional cattle, goats and sheep reared in livestock-wildlife interface areas of Zambia. Tropical Animal Health and Production 38: 195-206

14. OIE 2006 Handistatus II 1996-2004 http:// www.oie.int/hs2/sit_mald_cont.asp?c_ mald $=33 \&$ c cont $=1$

15. Paweska J T, Potts A D, Harris H J, Smith S J, Viljoen G J, Dungu B, Brett O L, Bubb M, Prozesky L 2002 Validation of an indirect enzyme-linked immunosorbent assay for the detection of antibody against Brucella abortus in cattle sera using an automated ELISA workstation. Onderstepoort Journal of Veterinary Research 69: 61-77

16. Qazilbash A A, Bari A, 1997 Sero-diagnosis of human brucellosis among TB suspected patients. Journal of the Pakistan Medical Association 47: 243-246

17. Rogan W J, Gladen B, 1978 Estimating prevalence from the results of a screening test. American Journal of Epidemiology 107: 71-76

16. Salman M D, Meyer M E 1984 Epidemiology of bovine brucellosis in the Mexicali Valley, Mexico: literature review of diseaseassociated factors. American Journal of Veterinary Research 45: 1557-1560

19. Stemshorn B W, Forbes L B, Eaglesome MD, Nielsen KH, Robertson F J, Samagh BS 1985 A comparison of standard serological tests for the diagnosis of bovine brucellosis in Canada. Canadian Journal of Comparative Medicine 49: 391-4

20. Thrusfield M 2005 Veterinary epidemiology (3rd edn). Blackwell Science, London: 245 